\title{
Relationship between cerebral blood flow and the development of swelling and life-threatening herniation in acute ischemic stroke
}

Andrew D. Firlik, M.D., Howard Yonas, M.D., Anthony M. Kaufmann, M.D., Lawrence R. Wechsler, M.D., Charles A. Jungreis, M.D., Melanie B. Fukui, M.D., and Robert L. Williams, M.D. Departments of Neurological Surgery, Neurology, and Radiology, University of Pittsburgh Medical Center, Pittsburgh, Pennsylvania

Object. The purpose of this study was to determine whether cerebral blood flow (CBF) measurements in acute stroke could be correlated with the subsequent development of cerebral edema and life-threatening brain herniation.

Methods. Twenty patients with aggressively managed acute middle cerebral artery (MCA) territory strokes who underwent xenon-enhanced computerized tomography (Xe-CT) CBF scanning within 6 hours of onset of symptoms were retrospectively reviewed. The relationship among CBF and follow-up CT evidence of edema and clinical evidence of brain herniation during the 36 to 96 hours following stroke onset was analyzed.

Initial CT scans displayed abnormal findings in 11 patients (55\%), whereas the Xe-CT CBF scans showed abnormal findings in all patients $(100 \%)$. The mean CBF in the symptomatic MCA territory was $10.4 \mathrm{ml} / 100 \mathrm{~g} /$ minute in patients who developed severe edema compared with $19 \mathrm{ml} / 100 \mathrm{~g} / \mathrm{minute}$ in patients who developed mild edema ( $\mathrm{p}<0.05)$. The mean CBF in the symptomatic MCA territory was $8.6 \mathrm{ml} / 100 \mathrm{~g} /$ minute in patients who developed clinical brain herniation compared with $18 \mathrm{ml} / 100$ $\mathrm{g} /$ minute in those who did not $(\mathrm{p}<0.01)$. The mean CBF in the symptomatic MCA territory that was 15 $\mathrm{ml} / 100 \mathrm{~g} /$ minute or lower was significantly associated with the development of severe edema and herniation $(\mathrm{p}<0.05)$.

Conclusions. Within 6 hours of acute MCA territory stroke, Xe-CT CBF measurements can be used to predict the subsequent development of severe edema and progression to clinical life-threatening brain herniation. Early knowledge of the anatomical and clinical sequelae of stroke in the acute phase may aid in the triage of such patients and alert physicians to the potential need for more aggressive medical or neurosurgical intervention.

Key Words * cerebral blood flow * cerebral ischemia * stroke * computerized tomography * xenon study

Incidences of morbidity and mortality from nonhemorrhagic stroke relate to the degree of ischemia, duration of insult, and specific volume of brain affected. With the ongoing expansion of treatment 
options for acute ischemic stroke, the need to characterize the prognostic features of a cerebral ischemic event rapidly and accurately is critical.[1,2,4,13-15,17,23] Although useful in excluding hemorrhage as a cause, computerized tomography (CT) scanning frequently displays normal findings or reveals only subtle changes in patients suffering from acute ischemic stroke.[5,24,26-29] Functional CT cerebral blood flow (CBF) measurements with xenon enhancement (Xe-CT), however, can define the location, volume, and degree of ischemia.[10,12,32-35] The purpose of this study was to determine, for the first time, whether acute CBF measurements in patients with ischemic stroke could be correlated with the subsequent development of edema and life-threatening brain herniation in a population of aggressively managed patients with nonhemorrhagic strokes in the anterior circulation.

\section{CLINICAL MATERIAL AND METHODS}

\section{Patient Population}

Twenty patients who were admitted to the University of Pittsburgh Medical Center with the clinical diagnosis of acute middle cerebral artery (MCA) territory stroke were studied retrospectively. A total of 56 patients who underwent Xe-CT scanning from June 1996 to June 1997 were initially reviewed. Patients were included if they had: 1) acute MCA territory stroke symptoms consisting of, but not limited to, severe hemiparesis or hemiplegia and ipsilateral sensory loss with or without aphasia or neglect; 2) a modified National Institutes of Health Stroke Scale[21] score of 15 or higher; 3) a CT scan that excluded hemorrhage as a cause; and 4) an Xe-CT scan that had been obtained within 6 hours of symptom onset. (Xe-CT scans are standard diagnostic studies used at our institution in the evaluation of patients with suspected cerebral ischemia.) Patients with lacunar syndromes, events related to the vertebrobasilar system, isolated visual complaints, or resolving deficits were excluded. One patient was excluded because his Xe-CT study was technically inadequate. The results of the Xe-CT scans were not known at the time of selection of patients for inclusion in this study and, therefore, did not influence the decision to include or exclude patients. Patients varied in age from 26 to 84 years (mean 66 years); there were nine men $(45 \%)$ and 11 women (55\%). Fourteen patients presented with left hemispheric events (70\%) and six (30\%) with right hemispheric events.

\section{Blinded Interpretation of Initial CT Scans}

All patients underwent noncontrast-enhanced CT scanning within 6 hours of the onset of symptoms (GE HiSpeed Scanner; General Electric Medical Systems, Milwaukee, WI). The mean interval between the onset of symptoms and CT scanning was 230 minutes. All CT scans were independently evaluated by two fellowship-trained neuroradiologists who were informed only that the CT scans were those of patients who were clinically suspected to have had a stroke. The neuroradiologists were blinded to: 1 ) specific stroke symptoms and the involved side; 2) CBF data; and 3) angiographic findings. The CT images were evaluated for the presence or absence of hypodensity (cortical or basal ganglia), mass effect (sulcal or ventricular effacement), and hyperdensity in the MCA. Differences were adjudicated by consensus between the two neuroradiologists.

\section{Xenon-Enhanced CT CBF Testing}

All patients underwent Xe-CT CBF scanning immediately following initial CT scanning within 6 hours from the onset of symptoms and before initiation of any stroke therapy. Quantitative Xe-CT CBF studies were performed using CT scanners to which an independent system for xenon delivery and CBF calculation had been added (Xe/CT System; Diversified Diagnostic Products, Houston, TX). Patients 
inhaled a 33\% Xe/67\% $\mathrm{O}_{2}$ mixture (XeScan; Praxair Pharmaceutical Gases, Danbury, CT) for 4.5 minutes, during which CT scans were obtained at three levels through the brain. Setup and computer calculation time required approximately 10 additional minutes, making the total time required to obtain CBF images approximately 15 minutes.

\section{Interpretation of Xe-CT CBF Scans}

The Xe-CT CBF scans were interpreted using a data analysis computer software program that determines the mean CBF within a series of 2-cm circular regions of interest (ROIs) distributed throughout the cortical and subcortical areas (Fig. 1).

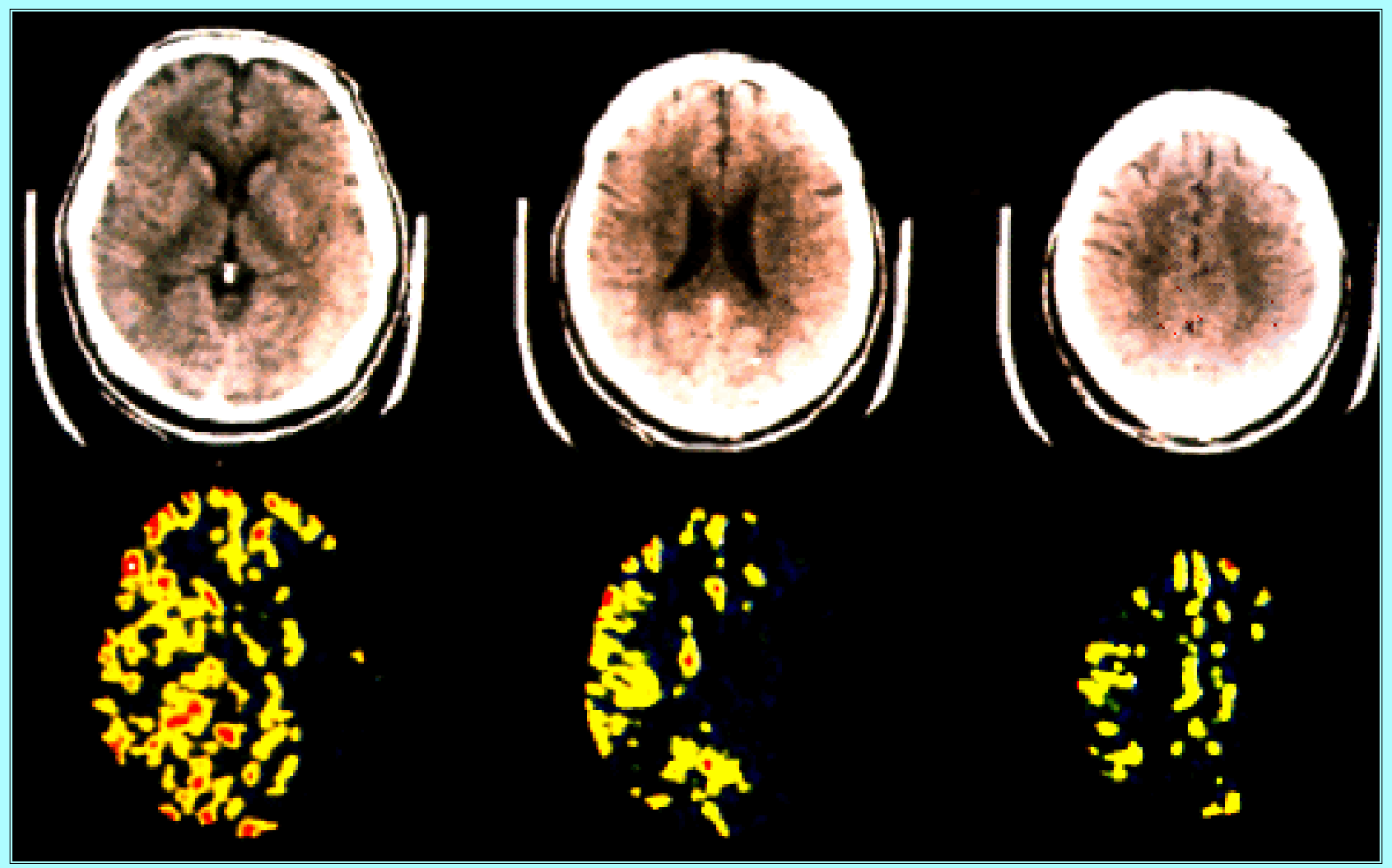

Fig. 1. Computerized tomography scans (upper) and corresponding Xe-CT CBF maps (lower) through three levels of brain in a patient who presented 3 hours after onset of right-sided hemiparesis and aphasia. The CT scans show a subtle left frontal hypodensity; however, the Xe-CT CBF maps show that the left MCA territory has markedly decreased perfusion with a CBF or $15 \mathrm{ml} / 100 \mathrm{~g} /$ minute or lower throughout. During the subsequent 48 hours, this patient developed severe edema and uncal herniation.

Three axial CT scan slices were studied, yielding between 55 and 65 ROIs per patient (Fig. 2). Approximately 1200 ROIs obtained in the study group were analyzed in this manner. The ROIs within areas that corresponded to artifacts on CT scans were excluded from the analysis. The mean CBF in the MCA territories was calculated based on the mean value of all ROIs in the region most commonly supplied by the MCA territory on each of the three CBF images obtained (Fig. 2). 


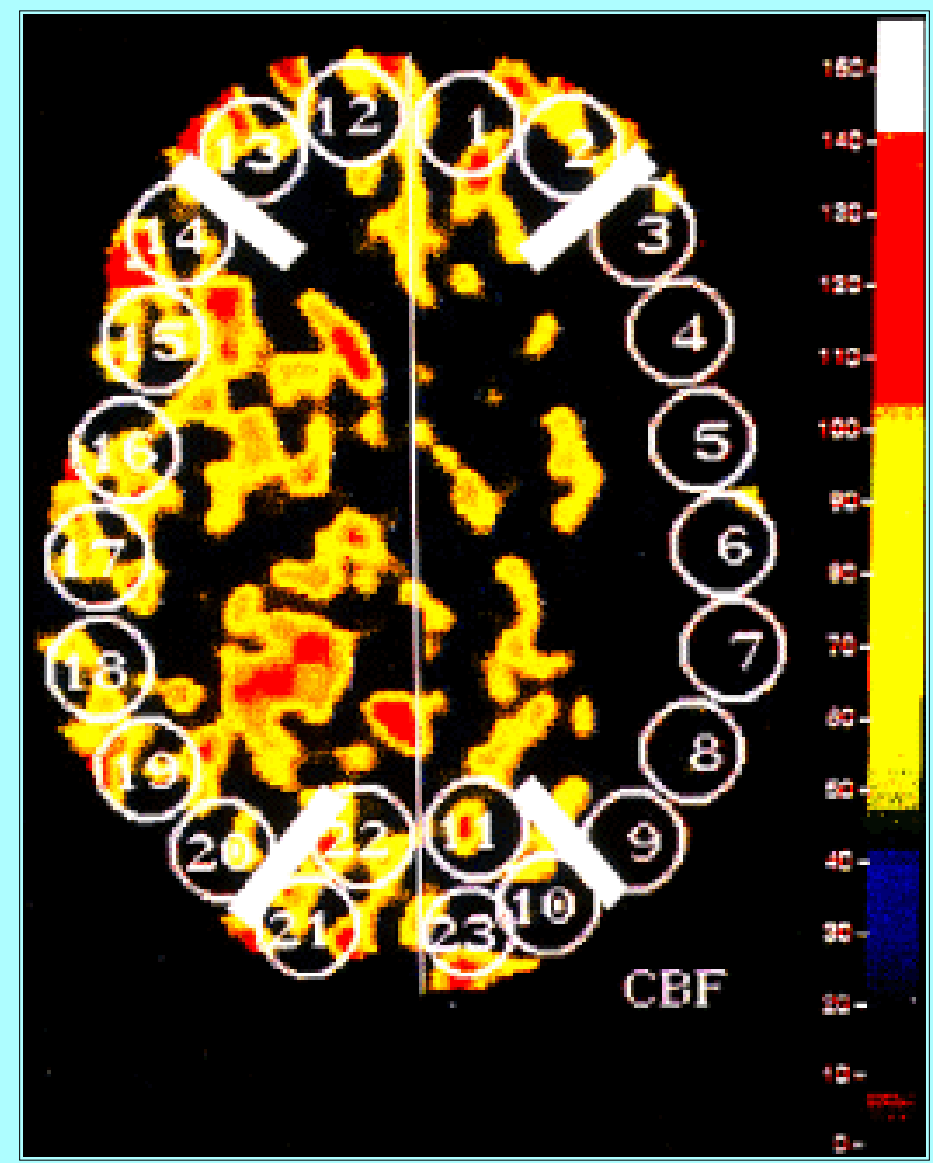

Fig. 2. Xenon-enhanced CT CBF map obtained in the same patient shown in Fig. 1 demonstrating detail of a single CT scan slice with superimposed ROIs used for calculating mean $\mathrm{CBF}$ and associated color coding scale (units are milliliters per $100 \mathrm{~g}$ per minute). The MCA territories are shown between the two white bars in both hemispheres.

\section{Patient Management}

All patients were admitted to a neurological intensive care unit and aggressively managed medically. Based on individual physician preferences and decisions on the part of patients and guardians, 11 (55\%) of the 20 patients underwent transfemoral angiography as a precursor to undergoing intraarterial thrombolysis within 6 hours of symptom onset (mean time to angiography 3 hours and 26 minutes; range 55 minutes-5 hours and 25 minutes). Ten patients (50\%) received intraarterial urokinase. One patient $(5 \%)$ received either prourokinase or a placebo (as part of a separate study protocol). Of the group of 11 patients who underwent angiography, in one patient an occluded $\mathrm{M}_{1}$ segment was believed to be successfully reperfused (complete recanalization achieved) and in five patients with $\mathrm{M}_{1}$ occlusions or combined internal carotid artery- $\mathrm{M}_{1}$ occlusions partial success was achieved, with only a minor degree of recanalization and reperfusion in occluded vessels. No success in reperfusion was achieved in the remaining five patients who underwent angiography that disclosed $\mathrm{M}_{1}$ or combined internal carotid artery- $\mathrm{M}_{1}$ occlusions. All patients were treated byadministration of heparin immediately following angiography unless conversion to hemorrhagic stroke was identified.

The remaining nine patients (45\%) who did not undergo angiography were treated with intravenous heparin anticoagulation therapy. Patients were not treated with a prophylactic course of mannitol, diuretic or corticosteroid medication, or hyperventilation; mannitol was administered if signs of herniation developed. 


\section{Clinical Assessment}

Patients were treated medically in a neurological intensive care or intermediate care ("step-down") unit and serial neurological examinations were recorded. Uncal herniation was noted if a patient developed pupillary irregularity and/or enlargement ipsilateral to the stroke in association with a decline in consciousness and development of extremity posturing.

\section{Follow-Up CT Scans}

Follow-up CT scans were obtained in 17 patients during the period of maximal cerebral swelling that corresponded to the patients' worst clinical condition (36-96 hours) following their initial event. Three patients exhibited rapid and progressive clinical improvement following their initial event and, therefore, did not undergo follow-up CT scanning during this time window. The CT scans were evaluated for the presence of edema and associated midline shift. Those patients in whom the CT scans displayed less than $1 \mathrm{~cm}$ of midline shift of the pineal gland and no obliteration of the ipsilateral ambient cistern were labeled as having "mild edema," whereas patients in whom scans showed a midline shift of the pineal gland measuring $1 \mathrm{~cm}$ or more with or without obliteration of the ipsilateral ambient cistern were labeled as having "severe edema." Location and size of the parenchymal hemorrhages were recorded. The follow-up scans were not read in a blinded manner because of the objective criteria available for assessment.

\section{RESULTS}

\section{Initial CT Scan Findings}

Initial CT scans yielded normal findings in nine (45\%) of the 20 patients. In the 11 patients $(55 \%)$ with abnormal initial CT scans, all (100\%) showed cortical areas of hypodensity, four (36\%) showed areas of hypodensity in the basal ganglia, four (36\%) showed cortical mass effect, and four (36\%) showed areas of hyperdensity (thromboses) in the MCA. All abnormalities were found on the symptomatic side; no abnormalities were detected on the asymptomatic side.

\section{Comparison of CBF in Symptomatic and Asymptomatic MCA Territories}

In all patients (100\%) the Xe-CT scans displayed abnormal findings. There were ROIs with a mean $\mathrm{CBF}$ of $20 \mathrm{ml} / 100 \mathrm{~g} /$ minute or lower within the symptomatic MCA territories in all cases. The mean CBF in the symptomatic MCA territories for the group was $15.2 \mathrm{X} 8.1 \mathrm{ml} / 100 \mathrm{~g} /$ minute compared with $34.2 \mathrm{X}$ $9.2 \mathrm{ml} / 100 \mathrm{~g} /$ minute in the asymptomatic MCA territories, a statistically significant difference $(\mathrm{p}<$ 0.0005; two-tailed t-test). The mean CBF in the symptomatic MCA territory ranged from 4 to $37 \mathrm{ml} / 100$ $\mathrm{g} /$ minute among individual patients.

\section{Relationship Between CBF and Edema on Follow-Up CT Scans}

On follow-up CT scans, mild edema was observed in eight patients and severe edema in nine patients. (The three patients who improved rapidly with no period of clinical deterioration that would suggest edema formation and mass effect did not undergo CT scanning during the 36- to 96-hour time window.) The mean $\mathrm{CBF}$ in the symptomatic MCA territories in the group of patients who developed mild edema was 19 X $8.2 \mathrm{ml} / 100 \mathrm{~g} /$ minute compared with $10.4 \mathrm{X} 6.2 \mathrm{ml} / 100 \mathrm{~g} / \mathrm{minute}$ for the group of patients who had severe edema, a significant difference ( $\mathrm{p}<0.05$; two-tailed t-test) (Fig. 3). The mean CBF in the symptomatic MCA territory in patients in whom follow-up CT scans were not obtained was 24.3 X 6.7 
$\mathrm{ml} / 100 \mathrm{~g} /$ minute. There was no significant difference between mean CBF in the MCA territories of the asymptomatic control hemispheres in patients with mild edema $(34.3 \times 7.9 \mathrm{ml} / 100 \mathrm{~g} /$ minute $)$ compared with patients with severe edema (33.2 X $7 \mathrm{ml} / 100 \mathrm{~g} /$ minute).

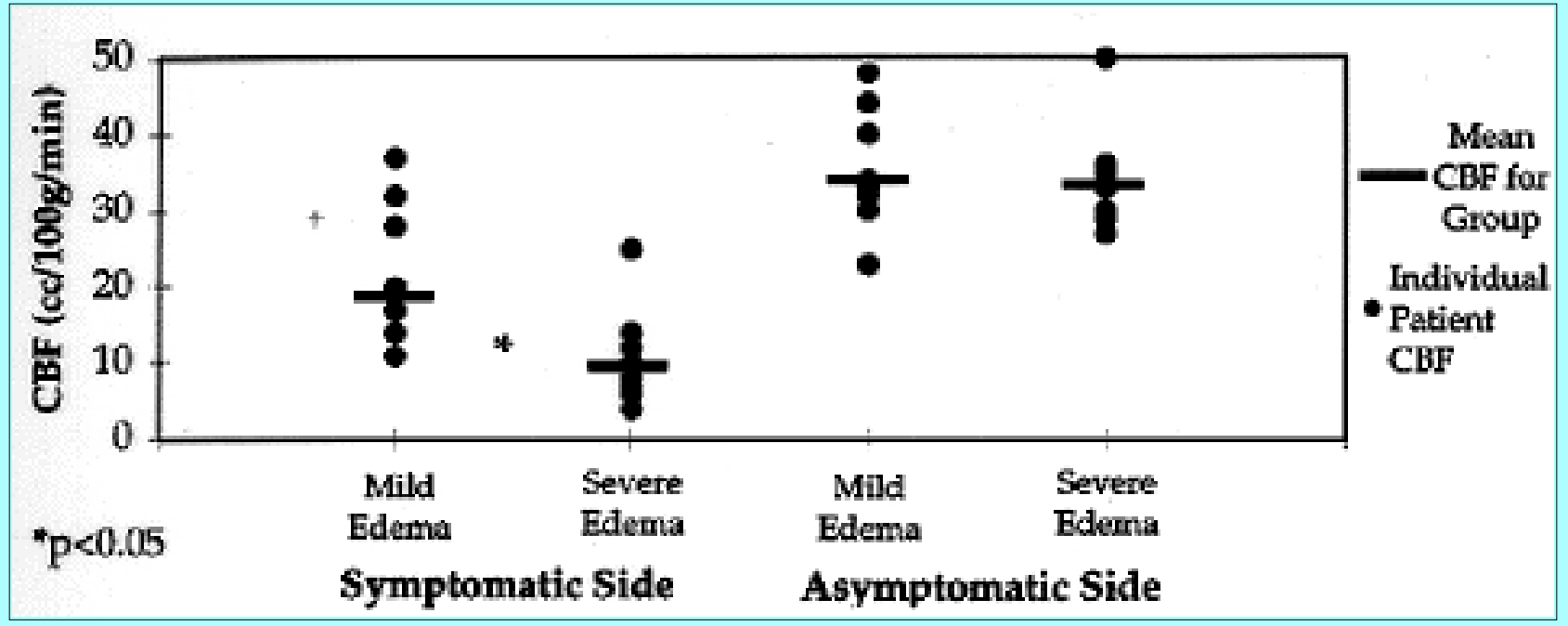

Fig. 3. Graph showing a comparison of MCA territory $\mathrm{CBF}$ in patients with mild edema and those with severe edema on follow-up CT scans.

Severe edema developed in eight (73\%) of 11 patients with a mean CBF of $15 \mathrm{ml} / 100 \mathrm{~g} /$ minute or lower in the symptomatic MCA territory, as observed on follow-up CT scans, compared with one (17\%) of six patients with a mean CBF higher than $15 \mathrm{ml} / 100 \mathrm{~g} /$ minute in the same location. Severe edema was 13.3 times more likely to occur in patients who had a mean CBF of $15 \mathrm{ml} / 100 \mathrm{~g} /$ minute or lower in the symptomatic MCA territory. The development of severe edema was significantly associated with a mean CBF of $15 \mathrm{ml} / 100 \mathrm{~g} /$ minute in the symptomatic MCA territory ( $\mathrm{p}<0.05$, Fisher's exact test). The sensitivity and specificity of the association between a mean MCA territory CBF of $15 \mathrm{ml} / 100 \mathrm{~g} / \mathrm{minute}$ or lower and severe edema formation is tabulated in Table 1.

\begin{tabular}{|c|c|c|}
\hline \multicolumn{3}{|c|}{$\begin{array}{l}\text { TABLE } 1 \\
\text { RELATONSHIP BETWEEN A MEAN CBF OF } 15 \mathrm{M} / 100 \text { GIMINUTE OR } \\
\text { LOWER IN THE AFFECTED MCA TERRITORY AND THE DEVELOPMENT } \\
\text { OF SEVERE EDEM AND HEFNIATION }\end{array}$} \\
\hline Statistical Factor & Severe Edema (\%) & Hemiation (\%) \\
\hline $\begin{array}{l}\text { Sensitivity } \\
\text { spedicity } \\
\text { positi ie predictive value } \\
\text { negative predictive value }\end{array}$ & $\begin{array}{l}89 \\
63 \\
73 \\
83\end{array}$ & $\begin{array}{r}100 \\
50 \\
46 \\
100\end{array}$ \\
\hline
\end{tabular}

\section{Relationship Between CBF and Clinical Herniation}

A clinical uncal herniation syndrome developed in six patients $(30 \%)$. The mean CBF in the symptomatic MCA territories in these patients was $8.6 \times 3.7 \mathrm{ml} / 100 \mathrm{~g} /$ minute compared with $18 \mathrm{X} 9.5 \mathrm{ml} / 100$ $\mathrm{g} /$ minute in patients who did not later show evidence of herniation, a significant difference $(\mathrm{p}<0.01$, two-tailed t-test) (Fig. 4). There was no significant difference between mean CBF in the asymptomatic control MCA territories in patients with herniation $(34.5$ X $8.4 \mathrm{ml} / 100 \mathrm{~g} /$ minute $)$ compared with patients without herniation (34 X $8.4 \mathrm{ml} / 100 \mathrm{~g} /$ minute). 


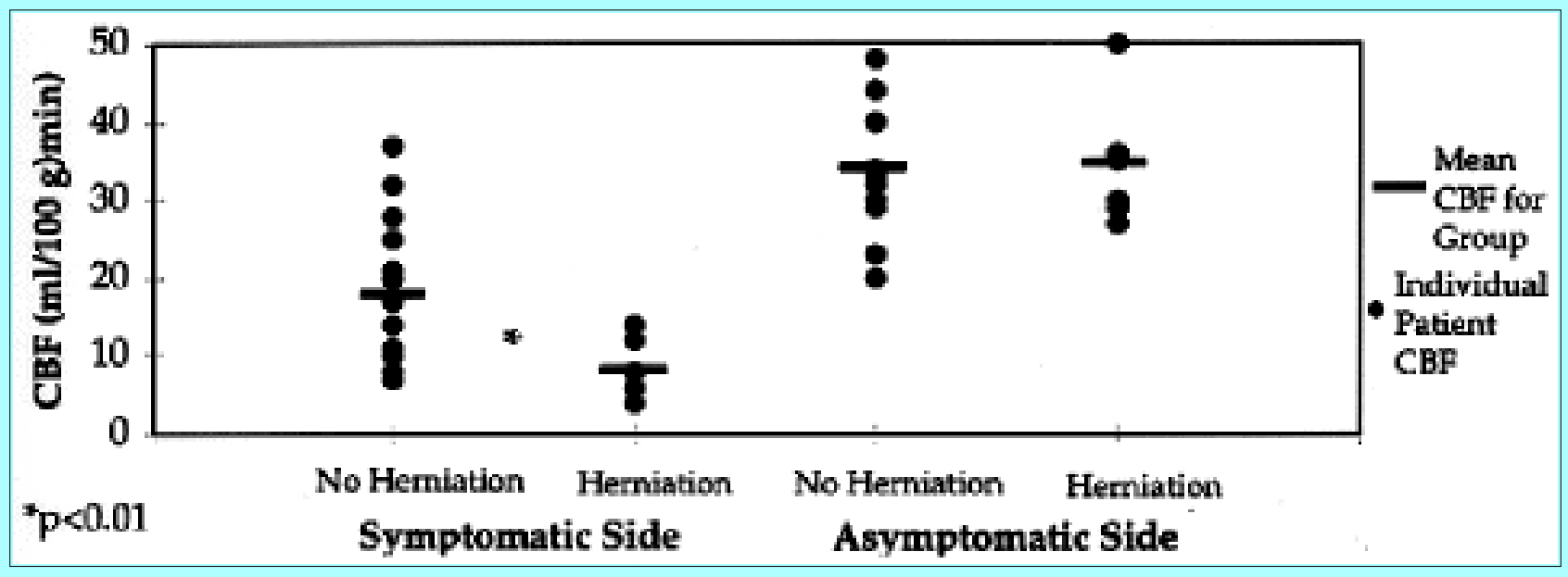

Fig. 4. Graph showing a comparison of MCA territory CBF in patients who developed life-threatening uncal herniation and those who did not.

Clinical herniation developed in six (46\%) of 13 patients with a mean CBF of $15 \mathrm{ml} / 100 \mathrm{~g} / \mathrm{minute}$ or lower in the symptomatic MCA territory, whereas no clinical herniation developed in the seven patients with a mean CBF higher than $15 \mathrm{ml} / 100 \mathrm{~g} /$ minute in the same location. All six patients (100\%) in whom life-threatening herniation developed had a mean CBF of $15 \mathrm{ml} / 100 \mathrm{~g} / \mathrm{minute}$ or lower in the symptomatic MCA territory and the development of life-threatening uncal herniation was significantly associated with this level of CBF in the symptomatic MCA territory ( $\mathrm{p}<0.05$; Fisher's exact test). The sensitivity and specificity of the association between a mean MCA territory CBF of $15 \mathrm{ml} / 100 \mathrm{~g} / \mathrm{minute}$ or lower and herniation is tabulated in Table 1.

\section{Relationship Between Swelling and Life-Threatening Herniation}

All six patients in whom uncal herniation developed exhibited severe edema on their follow-up CT scans. Among patients in whom herniation did not develop, eight (73\%) of 11 had mild edema. The development of severe edema was significantly associated with progression to uncal herniation $(\mathrm{p}<0.01$; Fisher's exact test).

\section{Relationship Between Early CT Findings and Development of Edema and Herniation}

There was no significant relationship between the presence of any abnormalities on the initial CT scans and the subsequent development of either edema or clinical herniation.

\section{Relationships Among CBF, Reperfusion, and Herniation}

There were no significant differences between mean CBF in either the symptomatic or asymptomatic MCA territories in patients who underwent attempted intraarterial thrombolytic therapy (11 patients) compared with those who did not (nine patients). Similarly, there were no significant differences in the initial mean $\mathrm{CBF}$ in the MCA territories of patients in whom reperfusion was either partially (five patients) or totally (one patient) achieved compared with those in whom reperfusion was either not achieved (five patients) or not attempted (nine patients). There was no significant relationship between reperfusion and the development of either severe edema or herniation.

\section{Relationships Among CBF, Hemorrhage, and Herniation}

Hemorrhagic conversion of MCA infarctions developed in three patients; all three were among the group that manifested clinical evidence of herniation. Two of these patients underwent attempted but only 
partially successful intraarterial thrombolysis; the third patient underwent angiography but no successful reperfusion was achieved. There was no significant association between mean CBF in the MCA territories and development of hemorrhage. Similarly, there was no significant relationship between development of hemorrhage and progression to herniation.

\section{DISCUSSION}

\section{Relationships Among CBF, Edema Formation, and Clinical Herniation}

This is the first study in which quantitative CBF measurements in patients with acute stroke have been reported in relation to subsequent development of edema formation and life-threatening brain herniation. In patients who presented within 6 hours of onset of symptoms a lower mean CBF in the symptomatic MCA territory was significantly associated with the development of severe edema and the uncal herniation syndrome. This correlation between diminished CBF and the development of edema and herniation has an important physiological basis: lower mean CBF in the MCA territory correlates with larger volumes of brain with zero or very low $\mathrm{CBF}$ that are destined to infarction irrespective of intervention.[8] The volume of brain that progresses to infarction would intuitively be expected to correlate with a greater degree of edema formation and an increased likelihood of progression to herniation. In this study, a mean CBF in the affected MCA territory that was $15 \mathrm{ml} / 100 \mathrm{~g} /$ minute or lower was an absolute requirement for herniation, signifying the large volume of brain parenchyma with very low $\mathrm{CBF}$ necessary to produce swelling sufficient to cause herniation. Early areas of hypodensity seen on CT scans, although representing early evidence of edema formation, were not significantly associated with the subsequent development of edema or herniation. Although an early area of hypodensity on a CT scan has been shown to have some prognostic significance in cases of acute stroke,[31] the fact that none was seen in this small group of patients further emphasizes the superior prognostic sensitivity of CBF measurements in this setting.

\section{Diagnosis of Acute Ischemic Stroke Achieved Using CBF Measurements}

Single-photon emission CT (SPECT)[3,7,11,31] and ${ }^{133} \mathrm{Xe}$ techniques[16] have been used for CBF measurements in acute stroke, however, they have had only limited utility because of poor spatial resolution and lack of quantitative accuracy. Newer magnetic resonance imaging diffusion and perfusion weighting techniques $[9,18,25,30]$ have allowed for identification of early cerebral ischemia, but provide only inferences about CBF based on assessments of cerebral blood volume. Positron emission tomography measurements of cerebral metabolism and CBF have also shown some predictive value in stroke assessment.[21] In this study, quantitative CBF measurements obtained using the Xe-CT method were highly sensitive and accurate in determining the ischemic origin of the acute neurological deficits seen in this population of patients, despite the fact that initial CT scans appeared to be abnormal in only $55 \%$ of patients. All patients had demonstrable regions of brain with decreased perfusion in the critical ischemic range ( $3 / 420 \mathrm{ml} / 100 \mathrm{~g} /$ minute) to explain their neurological deficits. The distinct advantage of using Xe-CT CBF studies in assessing acute stroke is that they are rapid, quantitative, and accurate[10,12,32-35] and can be obtained in conjunction with a CT scan, which would be performed routinely to rule out a hemorrhagic cause of the stroke. Thus, acquisition of quantitative CBF data by using Xe-CT scanning can be easily incorporated into an emergency stroke evaluation.

\section{Limitations of the Study}

In interpreting these results, one should consider the nature of the patient population studied. These 20 
patients all presented within 6 hours of onset of acute stroke symptoms and large, acute MCA territory strokes were initially diagnosed in each of them. All patients proved to have ischemia in the MCA territory that corresponded to their symptoms. Patients who had similar presentations that were explained by hemorrhagic, lacunar, or posterior fossa events, or those with resolving deficits were not included in this analysis. Therefore, the high sensitivity and specificity of the Xe-CT technique for detecting ischemia and the value of CBF measurements in predicting edema and herniation must be viewed in light of the specific population of stroke patients studied. It is likely that the large number of patients (six [30\%] of 20) in whom herniation progressed in this series of stroke patients represents the specific inclusion of patients with severe deficits on presentation who were believed to have significant degrees of MCA territory ischemia. This could have influenced the very high (100\%) sensitivity of CBF measurements in detecting ischemia in these patients; the sensitivity of the Xe-CT technique in detecting ischemia in a general stroke population will likely be lower. We do suspect, however, that CBF measurements will be even more predictive of severe edema formation and herniation when a more general stroke population is studied because patients with lesser degrees of ischemia (and much lower likelihood of severe edema formation and herniation) will be more easily differentiated from those with severely reduced CBF, such as those included in this study.

Despite the homogeneity of the inclusion criteria for the group, there were differences in the subsequent treatments that the patients received. Of the 11 patients who underwent attempted intraarterial thrombolysis, only one had complete angiographically confirmed reperfusion of an occluded $\mathrm{M}_{1}$ segment; five others had minor degrees of reperfusion, and in the remaining five the procedures were completely unsuccessful. The fact that various degrees of reperfusion were achieved in some patients is a potential source of bias in the study because the development of edema and herniation could have been partially caused by the interventions. This does not, however, appear to be true. It is important to note that there were no significant differences in initial CBF in any subgroup of these patients. Specifically, there was no difference in CBF between patients who underwent attempted intraarterial thrombolysis and those who did not. Similarly, there was no difference in CBF between patients in whom reperfusion was achieved and those in whom reperfusion was not achieved or not attempted. Therefore, initial differences in CBF do not appear to have created a bias for any subgroup of these patients. Furthermore, there was no significant association between reperfusion and the development of severe edema or herniation. One should note that, given the small number of patients in this study, although no statistically significant differences in these variables were seen, the lack of a relationship cannot be proven because of insufficient statistical power. A reasonable conclusion, nevertheless, is that lower CBF measurements can be associated with edema formation and herniation despite variably successful attempts at reperfusion by administration of intraarterial thrombolytic agents. However, a prospective study in which these issues could be addressed would be necessary to prove the predictive ability of Xe-CT scans in determining patient outcome.

\section{Implications for the Management of Acute Stroke}

We have provided evidence that quantitative CBF measurement in which Xe-CT scanning is used is a sensitive and accurate means of diagnosing acute ischemic stroke. Furthermore, the diagnostic information thus obtained has prognostic significance with respect to predicting the early clinical course of stroke patients and identifying those in whom there is a higher likelihood that severe edema and life-threatening brain herniation may develop despite intervention. Early knowledge of the anatomical and clinical sequelae of stroke in the acute phase may aid in the triage of such patients for stroke therapy 
and may help identify the potential need for more intensive medical and neurosurgical interventions. The ability to predict in which patients life-threatening herniation is more likely to develop irrespective of treatment will allow for earlier and less hurried discussions with patients and families about potentially life-saving emergency surgical procedures to protect remaining viable brain tissue, such as resection of infarcted cerebral tissue or decompressive hemicraniectomy operations.[6,19,20]

In addition to their prognostic value in the clinical care of individual stroke patients, CBF measurements may contribute substantially to stroke therapy research. At present, with the emphasis on very early intervention within hours of stroke onset, patients can only be assessed by clinical examination or conventional imaging, both of which provide a rough concept of the anatomical ischemic insult, but do not supply an understanding of its physiological severity. The efficacy of new pharmacological agents designed to alter the course of evolving infarction by reducing edema formation, for example, can be tested using CBF measurements as a means of determining the baseline severity of the ischemic events studied and the preintervention probabilities of subsequent edema formation or progression to herniation.

\section{Acknowledgments}

The authors thank John May for his management of the Xe-CT scan database and Susan Penthany and Lori Kirby for their involvement in the clinical care of patients undergoing Xe-CT scanning.

\section{References}

1. Anonymous: Thrombolytic therapy with streptokinase in acute ischemic stroke. Multicenter Acute Stroke Trial--Europe Study Group. New Engl J Med 335:145-150, 1996

2. Anonymous: Tissue plasminogen activator for acute ischemic stroke. The National Institute of Neurological Disorders and Stroke rt-PA Stroke Study Group. N Engl J Med 333:1581-1587, 1995

3. Brass LM, Walovitch RC, Joseph JL, et al: The role of single photon emission computed tomography brain imaging with $99 \mathrm{mTc}$-bicisate in the localization and definition of mechanism of ischemic stroke. $\mathbf{J}$ Cereb Blood Flow Metab 14 (Suppl 1):S91-S98, 1994

4. Brott TG, Haley EC Jr, Levy DE, et al: Urgent therapy for stroke. Part I. Pilot study of tissue plaminogen activator administered within 90 minutes. Stroke 23:632-640, 1992

5. Bryan RN, Levy LM, Whitlow WD, et al: Diagnosis of acute cerebral infarction: comparison of CT and MR imaging. AJNR 12:611-620, 1991

6. Carter BS, Ogilvy CS, Candia GJ, et al: One-year outcome after decompressive surgery for massive nondominant hemispheric infarction. Neurosurgery 40:1168-1176, 1997

7. Feldmann M, Voth E, Dressler D, et al: 99mTC-hexamethylpropylene amine oxime SPECT and x-ray CT in acute cerebral ischaemia. J Neurol 237:475-479, 1990

8. Firlik AD, Kaufmann AM, Wechsler LR, et al: Quantitative cerebral blood flow determinations in acute ischemic stroke. Relationship to computed tomography and angiography. Stroke 28:2208-2213, 1997

9. Fisher M, Prichard JW, Warach S: New magnetic resonance techniques for acute ischemic stroke. JAMA 274:908-911, 1995 
10. Fukui MB, Johnson DW, Yonas H, et al: Xe/CT cerebral blood flow evaluation of delayed symptomatic cerebral ischemia after subarachnoid hemorrhage. AJNR 13:265-270, 1992

11. Giubilei F, Lenzi GL, Di Piero V, et al: Predictive value of brain perfusion single-photon emission computed tomography in acute ischemic stroke. Stroke 21:895-900, 1990

12. Gur D, Good WF, Wolfson SK Jr, et al: In vivo mapping of local cerebral blood flow by xenon-enhanced computed tomography. Science 215:1267-1268, 1982

13. Hacke W, Kaste M, Fieschi C, et al: Intravenous thrombolysis with recombinant tissue plasminogen activator for acute hemispheric stroke: the European Cooperative Acute Stroke Study (ECASS). JAMA 274:1017-1025, 1995

14. Haley EC Jr, Brott TG, Sheppard GL, et al: Pilot randomized trial of tissue plasminogen activator in acute ischemic stroke. Stroke 24:1000-1004, 1993

15. Haley EC Jr, Levy DE, Brott TG, et al: Urgent therapy for stroke. Part II. Pilot study of tissue plaminogen activator administered 91-180 minutes from onset. Stroke 23:641-645, 1992

16. Halsey JH Jr, Nakai K, Wariyar B: Sensitivity of rCBF to focal lesions. Stroke 12:631-635, 1981

17. Hommel M, Boissel J, Cornu C, et al: Termination of trial of streptokinase in severe acute ischaemic stroke. MAST study group. Lancet 345:578-579, 1995 (Letter)

18. Hossmann KA, Hoehn-Berlage M: Diffusion and perfusion MR imaging of cerebral ischemia. Cerebrovasc Brain Metab Rev 7:187-217, 1995

19. Kalia KK, Yonas H: An aggressive approach to massive middle cerebral artery infarction. Arch Neurol 50:1293-1297, 1993

20. Kondziolka D, Fazl M: Functional recovery after decompressive craniectomy for cerebral infarction. Neurosurgery 23:143-147, 1988

21. Lyden P, Brott T, Tilley B, et al: Improved reliability of the NIH stroke scale using video training. Stroke 25:2220-2226, 1994

22. Marchal G, Serrati C, Rioux P, et al: PET imaging of cerebral perfusion and oxygen consumption in acute ischaemic stroke: relation to outcome. Lancet 341:925-927, 1991

23. Mori E, Tabuchi M, Yoshida T, et al: Intracarotid urokinase with thromboembolic occlusion of the middle cerebral artery. Stroke 19:802-812, 1988

24. Pressman BD, Tourje EJ, Thompson JR: An early CT sign of ischemic infarction: increased density in a cerebral artery. AJR 149:583-586, 1987

25. Sorensen AG, Buonanno FS, Gonzalez RG, et al: Hyperacute stroke: evaluation with combined multisection diffusion-weighted and hemodynamically weighted echo-planar MR imaging. Radiology 199:391-401, 1996

26. Tomsick T, Brott T, Barsan W, et al: Thrombus localization with emergency cerebral CT. AJNR 13:257-263, 1992 
27. Tomura N, Uemura K, Inugami A, et al: Early CT finding in cerebral infarction: obscuration of the lentiform nucleus. Radiology 168:463-467, 1988

28. Truwit CL, Barkovich AJ, Gean-Marton A, et al: Loss of the insular ribbon: another early CT sign of acute middle cerebral artery infarction. Radiology 176:801-806, 1990

29. von Kummer R, Meyding-Lamadé U, Forsting M, et al: Sensitivity and prognostic value of early CT in occlusion of the middle cerebral artery trunk. AJNR 15:9-18, 1994

30. Warach S, Gaa J, Siewert B, et al: Acute human stroke studied by whole brain echo planar diffusion weighted magnetic resonance imaging. Ann Neurol 37:231-241, 1995

31. Yokogami K, Nakano S, Ohta H, et al: Prediction of hemorrhagic complications after thrombolytic therapy for middle cerebral artery occlusion: value of pre- and post-therapeutic computed tomographic findings and angiographic occlusive site. Neurosurgery 39:1102-1107, 1996

32. Yonas H, Darby JM, Marks EC, et al: CBF measured by Xe-CT: approach to analysis and normal values. J Cereb Blood Flow Metab 11:716-725, 1991

33. Yonas H, Gur D, Claassen D, et al: Stable xenon enhanced computed tomography in the study of clinical and pathologic correlates of focal ischemia in baboons. Stroke 19:228-238, 1988

34. Yonas H, Gur D, Claassen D, et al: Stable xenon-enhanced CT measurement of cerebral blood flow in reversible focal ischemia in baboons. J Neurosurg 73:266-273, 1990

35. Yonas H, Sekhar L, Johnson DW, et al: Determination of irreversible ischemia by xenon-enhanced computed tomographic monitoring of cerebral blood flow in patients with symptomatic vasospasm.

Neurosurgery 24:368-372, 1989

Manuscript received January 12, 1998.

Accepted in final form March 24, 1998.

Dr. Yonas' work is partially supported by a research grant from Praxair, Inc.

Address reprint requests to: Howard Yonas, M.D., Department of Neurological Surgery, University of Pittsburgh Medical Center, 200 Lothrop Street, Suite B-400, Pittsburgh, Pennsylvania 15213. 